\title{
Drive power calculation of a crushing machine working in shear
}

\author{
Aleksandr Nikitin $^{1 *}$, Nikita Kurochkin ${ }^{1}$, and Yonghui Yang ${ }^{2}$ \\ ${ }^{1}$ Siberian State Industrial University, Novokuznetsk, 654007, Russia \\ ${ }^{2}$ University of Science and Technology Liaoning, Anshan, 14051, China
}

\begin{abstract}
The description of crusher design is given, in which the destruction of the processed material occurs due to the forces acting on the crushed piece in the same plane towards each other, while in the processed piece only tangential stresses arise. It makes possible to reduce the energy consumption per unit of finished product by almost two times.
\end{abstract}

\section{Introduction}

Energy consumption for crushing various materials makes up a large share (up to 5\%) in the energy balance of the world. Many industrial industries, including mining, process large quantities of bulk materials of various size classes. In most cases, the required size is achieved by crushing the pieces into fragments in crushers [1].

\section{Problem statement}

The power of the electric motor is determined taking into account the power expended to overcome all resistance forces during the operation of the crusher:

$$
N_{D R}=\frac{N_{1}+N_{2}}{\eta},
$$

where $N_{1}$ - the power required for crushing the material; $N_{2}$ - power expended on friction in the bearings; $\eta$ - transmission efficiency.

The calculation of power is carried out for a single act of crushing, that is, with the destruction of one original piece with subsequent correlation, taking into account the possibility of simultaneous crushing of several pieces.

One of the main indicators of the crushing process is the crushing energy efficiency determined by the mass of crushed material obtained by spending a unit of electricity. In industry, drillers are used to destroy fragile materials by compression (roll, jaw, conical) and impact (gear, hammer).

Compression crushers are divided into the following types: jaw crushers, in which destruction occurs due to the opposite working movement of the plates [2]; roller,

\footnotetext{
* Corresponding author: nikitin1601@yandex.ru
} 
destroying a piece when it is pulled into the gap between rollers rotating towards each other [3]; conical, in which crushing is carried out by compressing the material between the cones located with eccentricity one inside the other [4]. Crushing of brittle material in these crushers is carried out by compressive forces that create normal stresses in the piece, according to the limiting values of which their power is calculated. Crushers operating on the compression of the destroyed material have approximately the same specific energy intensity, that is, the same energy consumption for the destruction of a unit volume of material of equal strength [5]. Compression destruction is the most energy-intensive of the known crushing methods [6].

Impact (rotary and hammer) crushers are designed for impact crushing of various brittle materials using beaters rigidly fixed to a rotor rotating around a horizontal axis [7], while destruction occurs due to splitting.

The destruction of brittle rocks by impact and compression requires different energy inputs needed to destroy the material, while destruction by compression requires almost one and a half times the energy consumption compared to destruction by impact. However, hammer drills have a significant drawback, which is expressed in the fact that only $25-$ $30 \%$ of the finished product is obtained with a given fractional range [8].

Designs of single-roll crushers are known, in which a piece is fed into the gap between the roll and a solid stationary plate. In the process of work, a complex stress state is generated in the destroyed material, in which both compressive forces act on a piece of crushed material, causing the action of normal compressive stresses in the piece, and an internal torque that causes the action of tangential stresses [9]. Under the action of a complex stressed state in the material, the strength is estimated through the equivalent stress, the ultimate strength of which is less than under the action of only normal stresses [10]. A reduction in energy consumption for crushing is achieved, all other things being equal, by $20-30 \%$ in comparison with crushers operating in compression.

\section{Object of study}

In order to increase the energy efficiency of the process of brittle materials destruction, the Siberian State Industrial University developed a design of a rotary-cone crusher [11], in which crushing occurs due to the generation of only tangential stresses in a piece, under the action of which shear deformation occurs. With this type of deformation, the ultimate strength for a given material takes on the minimum possible value, equal to about 0.5 of the value of the ultimate strength in compression [12]. Hence, it follows that this crushing method is the most energy efficient in comparison with all others when obtaining a finished product of a given size.

In the process of operation of the crusher under consideration, the destruction of the processed material occurs due to the forces acting on the crushed piece in one plane towards each other, that is, the condition for the generation of only tangential stresses in the piece is provided.

The crusher consists of a body 1 made integral with a stationary cone and a rotating inner cone 2 located coaxially with it with a drive 3 (Fig. 1). The rotating inner cone is installed in radial bearings 5 and rests on a thrust bearing 4. On the working surfaces of the cones along their generatrices there are ribs 6 . In the upper part of the body 1 there is a feed chute 7 for material supply, and in the lower part there are discharge windows 8 .

Crushing occurs as follows. A piece of crushed material 9 through chute 7 is fed into the crushing zone formed by the working surfaces of the cones 1 and 2 . When the inner cone 2 rotates, the ribs 6 located on the working surfaces of the cones are periodically located opposite each other and form channels into which, under the action of gravitational forces, piece 9 falls to a depth at which its transverse size is equal to the size of the gap between 
the surfaces of the fixed outer and rotating inner cones 1 and 2 . With further rotation of the inner cone 2, piece 9 is clamped between the lateral surfaces of the ribs 6 and due to the occurrence of tangential stresses in it, shear deformation develops and the piece is destroyed.

The dimensions of the rib are characterized by the following parameters: the height of the rib $h$ and the angle of inclination of the side surface of the rib to the base $\alpha$. Their values are defined as follows. The height of the rib $h$ should not be more than 0.5 of the size of the gap between the surfaces of the stationary and rotating inner cones, in order to allow the inner cone to rotate. At $\alpha=90^{\circ}$, the fracture of the crushed piece occurs under the action of only shear stresses.

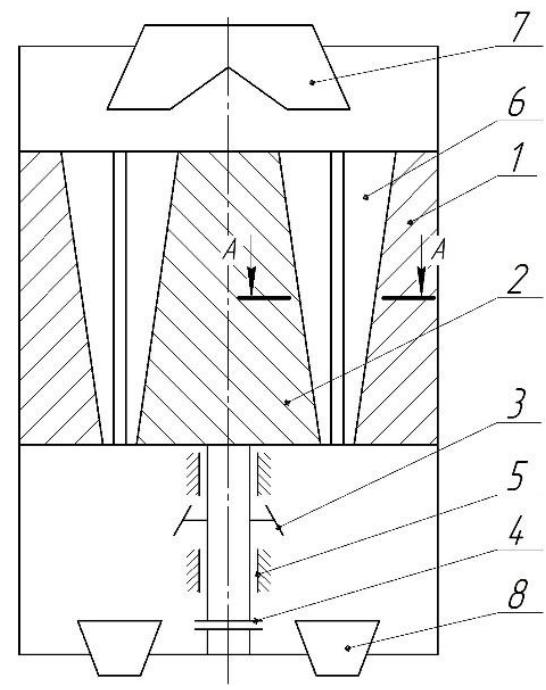

a
$\underline{A-A}$

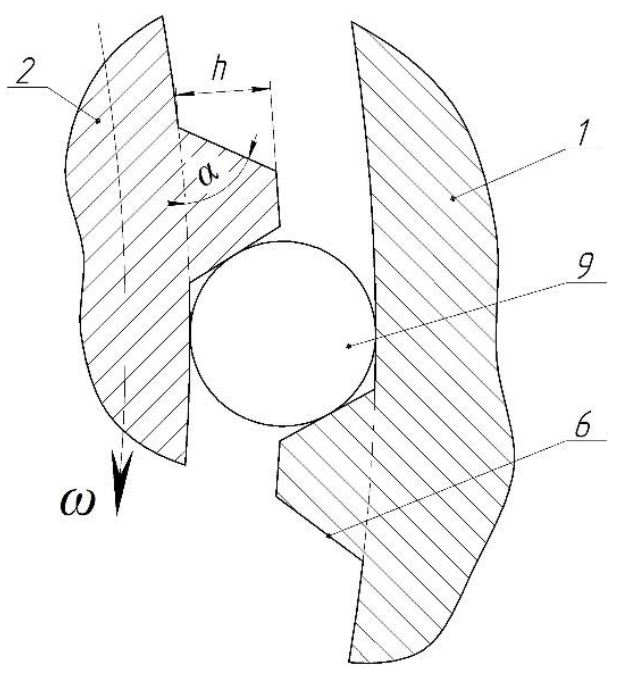

b

Fig. 1. Scheme of a rotary-cone crusher: $a$ - sectional view of the crusher; $b$ - section A-A.

The power spent on material crushing is consumed to overcome the moment of force $M$ :

$$
M=P . l,
$$

where $P$ - magnitude of the force required to carry out crushing; $l=R+h-$ moment arm $P$ relative to the center of the roll; $R$ - distance from the crusher axis to the base of the rib; $h-$ rib height, then the power required for crushing the material:

$$
N 1=M \cdot w,
$$

where $w$ is the angular velocity of inner cone rotation. The amount of force required to perform crushing

$$
P=\tau A,
$$

where $\tau$ is the magnitude of the shear stresses arising in the crushed piece; $A=\pi \cdot r^{2}-$ crosssectional area of the piece to be crushed; $r$ is the radius of the piece to be crushed. Therefore, taking into account expressions (3) and (4), the power required for crushing the material: 


$$
N_{l}=\tau \cdot A \cdot l \cdot w
$$

Power consumed to overcome the frictional force in the radial support bearing:

$$
N_{2}=\pi \cdot w \cdot d_{o} \cdot P \cdot f
$$

where $f$-coefficient of friction in the bearing; $d_{\mathrm{o}}$ - outer diameter of the bearing.

The joint solution of expressions (1), (5) and (6) makes it possible to calculate the power of the electric motor of a crushing machine operating in a shear:

$$
N_{d}=\frac{N_{1}+N_{2}}{\eta}=\frac{\tau \cdot A \cdot w}{\eta}\left(l+\pi \cdot d_{o} \cdot f\right) .
$$

Considering that the magnitude of the shear stresses is determined by the ratio:

$$
\tau=0.5 \cdot \sigma_{\text {com }}
$$

It can be concluded that energy consumption will be reduced by $50 \%$ compared to compression crushers.

Thus, the design of crushers, in which the destruction of the processed material occurs due to the generation of only tangential stresses in the piece, makes it possible to reduce the energy consumption per unit of finished product by almost two times.

\section{Conclusion}

The description of the crusher design is given, in which the destruction of the processed material occurs due to the forces acting on the crushed piece in one plane towards each other, while only tangential stresses arise in the processed piece. The use of crushers, in which the destruction of the processed material occurs due to the generation of only tangential stresses in the piece, makes it possible to reduce the energy consumption per unit of finished product by almost half.

\section{References}

1. B.V. Klushantsev, A.I. Kosarev, Yu.A. Muizemnek, Crushers (1990)

2. Telsmith. Jaw-crushers, www.telsmith.com

3. E.A.P Egbe, O.A. Olugboji, IJETT, 35, 511-515 (2016)

4. M. Johanssson, J. Quist, et al., Minerals Eng., 103-104, 93-101 (2017)

5. A.G. Nikitin, D.F. Sakharov, Izv. Vuzov. Fer. Metall., 4, 56-57 (2011)

6. V.A. Maslennikov, Izv. Vuzov. Mining Journal, 10-11, 124-138 (1996)

7. L.L. Zhao, F. Zang, et al., J. of China Univ. of Mining and Tech., 18, 316-320 (2008)

8. M.A. Fishman, Impact crushers (1960)

9. A.G. Nikitin, Yu.A. Epifantsev, et al., Izv. Vuzov. Fer. Metall., 62, 303-307, (2019)

10. J. Goulet, Resistance des materiaux (1976)

11. A.G. Nikitin, et al., RF Patent No. 2526738, Rotary crushing device (2014)

12. P.A. Stepin, Resistance of materials (2014) 